\title{
Factors Affecting Quality of Life Among Spinal Cord Injury Patients in Korea
}

\author{
Jong Soo Lee ${ }^{1}$, Sang Woon Kim¹, Sang Hyun Jee ${ }^{1}$, Joon Chul Kim², Jong Bo Choi ${ }^{3}$, Sung Yong Cho ${ }^{4}$, Jang Hwan Kim¹; Korea \\ Spinal Cord Injury Association \\ ${ }^{1}$ Department of Urology, Yonsei University College of Medicine, Seoul, Korea \\ ${ }^{2}$ Department of Urology, The Catholic University of Korea College of Medicine, Seoul, Korea \\ ${ }^{3}$ Department of Urology, Ajou University College of Medicine, Suwon, Korea \\ ${ }^{4}$ Department of Urology, Seoul National University College of Medicine, Seoul, Korea
}

Purpose: Complaints from spinal cord injury (SCI) patients are typically related to physical disability affecting activities of daily life. However, difficulties with voiding and defecation and/or sexual function can also be major concerns. The general population and even physicians are generally unaware of these complaints; therefore, this study focuses on surveying SCI patients regarding challenges that are faced in daily life.

Methods: A questionnaire was administered randomly and anonymously to SCI patients who visited the Korea Spinal Cord Injury Association and several rehabilitation hospitals in the Republic of Korea in 2013. All participants gave their consent prior to filling out the questionnaire.

Results: A total of 299 patients answered the questionnaire; the male to female ratio was 5.8:1 and common vectors for injury were motor vehicle accidents, industrial accidents, and falling down. Of the 169 patients who answered the 'most troublesome' and 'wish would improve' complaints questionnaire properly, urinary problems were most common, specifically incontinence and urinary tract infection. Among all patients, $67 \%$ were using clean intermittent catheterization, $63 \%$ were taking voidingrelated medications, $83 \%$ had sexual desires, and among the 122 patients who did not have offspring, $27 \%$ had future plans for children.

Conclusions: From this questionnaire, we discovered that SCI patients in the South Korea suffer not only from physical disability but also many other quality-of-life-related problems. When managing SCI patients, physicians should show greater concern and educate patients about problems related to voiding and sexual activity, rather than just physical disability.

Keywords: Spinal Cord Injuries; Urination Disorders; Sexual Behavior; Quality of Life; Surveys and Questionnaires

- Conflict of Interest: No potential conflict of interest relevant to this article was reported.

\section{INTRODUCTION}

Spinal cord injury (SCI) is roughly estimated to afflict a total of 74,000 patients in the South Korea, and that number is increasing by 2,000 each year [1]. In the United States, approximately
273,000 SCIs were recorded up to 2013, and that number is increasing by nearly 12,000 new patients each year [2]. From a nondisabled person's point of view, the chief complaint of a SCI patient would appear as loss of physical abilities or being unable to walk. However, in addition to physical disabilities, com-

Corresponding author: Jang Hwan Kim (iD http://orcid.org/0000-0002-9056-7072 Department of Urology, Yonsei University College of Medicine, 50-1 Yonsei-ro, Seodaemun-gu, Seoul 03722, Korea

E-mail: JKIM@yuhs.ac / Tel: +82-2-2228-2310/ Fax: +82-2-312-2538

Submitted: February 19, 2016 / Accepted after revision: April 21, 2016

(c) (i) This is an Open Access article distributed under the terms of the Cre(c) ${ }_{\mathrm{BY}} \mathrm{NC}_{\mathrm{NC}}$ ative Commons Attribution Non-Commercial License (http://creativecommons.org/licenses/by-nc/4.0/) which permits unrestricted non-commercial use, distribution, and reproduction in any medium, provided the original work is properly cited. 
plaints about problems with urination and defecation can also be major concerns for SCI patients. Concerns about giving birth and sexual desires are also present, although in South Korea they are not readily expressed due to adherence to the nation's Confucian-based values emphasizing modesty. Unfortunately, a lack of awareness of these complaints is not only common among the general public but also among physicians [3,4]. Due to a shortage of research on this topic, the current study surveyed SCI patients and attempted to outline symptoms and causes of discomfort in their daily lives.

\section{MATERIALS AND METHODS}

\section{Patients}

Data were collected via a survey administered randomly and anonymously to SCI patients who visited the Korea Spinal Cord Injury Association or several rehabilitation hospitals throughout Korea in 2013. Consent was obtained from all participants

\section{Questionnaire}

Thirty questions were designed consisting of 4 major categories: basic information, SCI-related discomforts, daily management of voiding, and sex life and family planning. The basic information category included 7 questions about age, sex, cause of injury, year of injury, period of admission, number of hospital admissions, and cohabitation status. The SCI-related category included 2 questions about patient discomfort: which ailment is most bothersome and which one the patient would most want to improve. The daily management of voiding category included 12 questions regarding current voiding method, advised voiding method, reason of not using advised method, voiding-related complications, source of urogenital information, recent urologic evaluation, cost of management for voiding in 2013, urologic medication, etc. Finally, the sex and family planning category included 9 questions about the presence of children, family plan, sex life, satisfaction and dissatisfaction with sex life, presence of sexual desire, and recommended and currently used supportive method for sexual activity (Supplementary material).

\section{Statistical Analysis}

Descriptive analyses were performed in Microsoft Excel. A oneway analysis of variance (ANOVA) was used for continuous variables in subgroup analysis. After ANOVA, a Bonferroni post hoc test was used for comparison between groups. For categorical variables, Pearson chi-square test was used. Statistical analysis was performed using IBM SPSS Statistics ver. 23.0 (IBM Co., Armonk, NY, USA), and statistical significance was set at $\mathrm{P}<0.05$.

\section{RESULTS}

\section{Demographic Characteristics and Injury-Related Data}

A total of 299 SCI patients were surveyed in 2013. The male to female ratio was 5.8:1, and the average age, age at time of injury, and disease duration were not significantly different according to sex. SCI was mostly caused by traumatic causes (91.4\%), among which motor vehicle accidents were most common, followed by falls and industrial accidents. Common nontraumatic causes included myelitis, tumor, and spina bifida. Injury rates according to sex are summarized in Table 1.

The average hospitalization period per admission was 7.6 months, and the average number of hospital admissions was 3.1 hospitals per patient. Patients were shown to live with spouses (42\%), parents (27\%), alone (25\%) and other (2\%). There was no significant difference between average hospitalization period and whom the patient was living with $(\mathrm{P}=0.97)$.

\section{'Most Troublesome' and 'Wish Would Improve' Complaints Among SCI Patients}

In the survey, responders were asked to rank their bothersome problems and those which they would most want to be im-

Table 1. Basic information of responders

\begin{tabular}{lcccc}
\hline Variable & Male & Female & No. (\%) & p-value \\
\hline No. of patients & 255 & 44 & & - \\
Age at survey (yr) & $46.4 \pm 10.3$ & $46.7 \pm 12.7$ & & $0.934^{\text {a) }}$ \\
Age at injury (yr) & $31.4 \pm 10.1$ & $30.9 \pm 12.1$ & & $0.821^{\text {a) }}$ \\
Disease duration (yr) & $14.8 \pm 9.1$ & $15.8 \pm 13.0$ & & $0.700^{\text {a) }}$ \\
Cause of SCI & 249 & 43 & 292 & $0.050^{\text {b) }}$ \\
Traumatic & 231 & 36 & $267(91.4)$ & \\
Nontraumatic & 18 & 7 & $25(8.6)$ & \\
Cause of traumatic SCI & 231 & 36 & 267 & $0.007^{\text {b) }}$ \\
Motor vehicle accident & 106 & 25 & $131(49.1)$ & \\
Industrial accident & 59 & 3 & $62(23.2)$ & \\
Sports & 6 & 0 & $6(2.2)$ & \\
Diving & 7 & 0 & $7(2.6)$ & \\
Falling down & 53 & 7 & $60(22.5)$ & \\
Violence & 0 & 1 & $1(0.4)$ & \\
\hline
\end{tabular}

Values are presented as mean \pm standard deviation or number. SCI, spinal cord injury.

${ }^{\text {a) }}$ Based on 2 sample $t$-test. ${ }^{\text {b) }}$ Based on Pearson chi-square test. 
Table 2. Ranking of 'most troublesome' and 'wish would improve' complaints of spinal cord injury patients

\begin{tabular}{lrrrrrrrrrrrr}
\hline \multirow{2}{*}{ Ranking } & \multicolumn{4}{c}{ Bothersome problems } & & \multicolumn{3}{c}{ Wish would improve } \\
\cline { 2 - 5 } \cline { 9 - 11 } & 1st & 2nd & 3rd & Total & & 1st & 2nd & 3rd & Total \\
\hline Physical disability & 132 & 21 & 15 & 168 & & 131 & 12 & 20 & 163 \\
Soreness & 36 & 41 & 14 & 91 & & 35 & 34 & 19 & 88 \\
Urologic problems & 66 & 97 & 35 & 198 & & 60 & 99 & 35 & 194 \\
Bowel problems & 28 & 74 & 59 & 161 & & 23 & 67 & 62 & 152 \\
Sexual activity & 6 & 20 & 50 & 76 & & 14 & 23 & 35 & 72 \\
Pain & 17 & 19 & 36 & 72 & & 20 & 22 & 34 & 76 \\
Hyper-reflexia & 6 & 10 & 33 & 49 & & 3 & 13 & 32 & 48 \\
Dyspnea & 0 & 1 & 6 & 7 & & 0 & 3 & 9 & 12 \\
Depression & 0 & 5 & 18 & 23 & & 1 & 5 & 10 & 16 \\
Other & 0 & 0 & 3 & 3 & & 0 & 1 & 2 & 3 \\
\hline
\end{tabular}

proved (Table 2). After excluding duplicate answers and nonresponses, complaints from 169 patients were reviewed. The most bothersome problem reported was physical disability, followed by urologic problems and bowel problems. Similarly, of the complaints that patients most wanted to be improved, first was physical disability; second was urinary problems; and third was bowel problems. However, when considering the 3 major reported complaints in order of frequency, urinary problems was the most frequently reported, followed by bowel problems and physical disability.

\section{Urinary Management}

The most commonly recommended methods for urination by physicians were clean intermittent catheterization (CIC) (47\%), followed by incontinent voiding (16\%), drugs (14\%), Foley catheterization ( $8 \%$ ), and cystostomy (4\%). However, about half of the patients were not using the recommended method because of economic problems (22\%), no effect (20\%), difficulty of use (20\%), no caregiver (14\%), side effects (10\%), and other reasons. Patients who were incontinent voiding used diapers or pads $(43 \%)$, gismos (31\%), and condoms or plastic bags $(21 \%)$. Other than these, percussion (36\%), the Crede method (29\%), and the Valsalva method (25\%) were also used. Only $15 \%$ of patients were using a single method for urination.

On average, patients experiencing incontinent voiding used a mean of 2.8 pads or diapers per day. Patients did CIC an average of 4.45 times per day, with a mean of $360 \mathrm{~mL}$ per time. For CIC materials, reusable silicon (56\%), disposable latex (28\%), and single-use silicon (11\%) were used. Although CIC was usu-
Table 3. Exposure to urogenital information

\begin{tabular}{lccc}
\hline & Doctors & Fellow patients & Internet \\
\hline $\begin{array}{l}\text { General complications } \\
(\mathrm{n}=313)\end{array}$ & $134(42.8 \%)$ & $162(51.8 \%)$ & $17(5.4 \%)$ \\
$\begin{array}{l}\text { Possibility for renal } \\
\text { injury }(\mathrm{n}=349)\end{array}$ & $186(53.3 \%)$ & $139(39.8 \%)$ & $24(6.9 \%)$ \\
\hline
\end{tabular}

ally performed by the patient (62\%), almost half of the patients required the assistance of a family member (20\%) or caregiver (16\%).

Regarding problems related to urination, incontinence (34\%) was the most commonly reported, followed by urinary tract infection (29\%), headache and dizziness (11\%), and bladder stones (4\%). Among patients using CIC, absence of a proper place $(26 \%)$ and side effects (26\%) were noted, followed by physical or economic burden (19\%), hygiene (15\%), and difficulty of use (12\%). Side effects included urinary tract infection (50\%), incontinence (15\%), headache and dizziness (12\%), and pain (10\%).

Regarding costs for urination management in 2013, 183 people responded that cost was about 90,000 Korean won (76 United States dollars) per month. A total of $63 \%$ of patients were taking drugs because of urinary tract problems such as an anticholinergic (43\%), antibiotic (25\%), or others (32\%).

\section{Exposure to Urogenital Information}

Via the survey, SCI patients were asked to report how they were informed of urogenital complications (e.g., doctors, fellow patients, internet; Table 3 ). There were no differences in current age $(\mathrm{P}=0.731)$, age at the time of injury $(\mathrm{P}=0.225)$, total length of hospital stay $(\mathrm{P}=0.902)$, or length of stay per hospital visit $(\mathrm{P}=0.521)$ according to where SCI patients received information on urogenital complications. Meanwhile, individuals who had a longer period of injury $(\mathrm{P}=0.02)$, and fewer hospitals visits $(\mathrm{P}=0.017)$ obtained information mainly from colleagues. Eighty percent of patients knew that bladder problems may accompany SCI leading to renal injury, and more than half of these patients received this information from doctors. Those who were informed about urogenital information by a doctor were also informed about the possibility of kidney damage by a doctor $(\mathrm{P}<0.001)$. Seventy-four percent of patients' most recent urinary tract evaluation was within the previous 3 years, with an average of 1.14 years. The length of time that had passed from the most recent urinary tract evaluation was significantly shorter in patients who received information from doctors 
Table 4. Offspring, plans for children, and sexual activity

\begin{tabular}{lcr}
\hline Offspring & Plans for children & Sexual activity \\
\hline Yes, $157(56 \%)$ & Yes, $9(6 \%)$ & Yes, $5(56 \%)$ \\
& No, $148(94 \%)$ & Yes, $39(26 \%)$ \\
No, 122 (44\%) & No, $109(74 \%)$ \\
& Yes, 33(27\%) & Yes, $9(27 \%)$ \\
& No, 89(73\%) & No, 24 $(73 \%)$ \\
& & Yes, $21(24 \%)$ \\
& & No, 68 (76\%) \\
\hline
\end{tabular}

about urination complications $(\mathrm{P}<0.001)$ and the possibility of kidney damage $(\mathrm{P}=0.004)$.

\section{Sexual Activity and Plans to Give Birth}

Among the study population, 56\% had offspring, and among those who did not have offspring, $27 \%$ were planning to have a child. Nevertheless, $73 \%$ of those planning for a child were not having intercourse (Table 4).

In the survey, SCI patients were asked to rank their sexual activity from 1 (very weak desire) to 5 (very strong desire). Patients who reported some desire for sexual activity (higher than 2) comprised $83 \%$, although of these patients only $28 \%$ were sexually active.

For the entire population, SCI patients were asked to rank their sexual satisfaction from 1 (very dissatisfied) to 5 (very satisfied). Patients who reported their satisfaction higher than average (greater than 3) comprised $25 \%$ of all patients, $22 \%$ of those with sexual desire, and $37 \%$ of those who were sexually active.

The most common complaints related to sexual function were erectile dysfunction (31\%) and lack of libido (27\%). Others included having no partner, short intercourse duration, and erection that did not lead to intercourse because of hospital stay. However, among all patients, 35\% received no recommendations about supportive methods for sexual activity. Lack of recommendations about supportive methods from physicians was reported by $34 \%$ of patients with plans for children and $29 \%$ of patients with sexual desire. Forty-seven percent of the patients with plans for children and $49 \%$ of patients with a sexual desire were not using supportive methods. When recommendations were given, $43 \%$ did not follow through because of financial burdens and $20 \%$ reported a lack of effect.

\section{DISCUSSION}

In South Korea, there are no statistics on SCI patients; even in determining the number of SCI patients in the country, one must rely on using foreign statistics [1]. For improvement of this problem and to build a database, an appropriate SCI model system, such as the US National Spinal Cord Injury Statistical Center (NSCISC), is needed [2,5].

In this study, the most common causes of SCI were traffic accidents and industrial accidents. While the total number of patients with traumatic SCI has been on the rise, the percentage of these common causes has also been increasing [6]. Prior to the 1970s, urinary problems were the most common cause of death, although recently this has changed to respiratory complications $[7,8]$. Previous studies have concluded that life expectancies post-SCI are increasing; in contrast, others have shown that survival during the first 2 years postinjury has improved, but long term survival has not $[9,10]$. However, due to the development of vehicle safety systems and emergency rescue techniques, immediate death after accident has been declining, which results in an increase of SCI patients $[8,11]$. In this situation, quality of life has become a criterion for outcome of SCI patients' management $[12,13]$. As shown above, secondary discomforts, which include voiding difficulty and problems with sexual activity, were shown to be some of the most bothersome and hoped-to-be-fixed problems in this study. To conclude, paying greater attention to patients' secondary complaints is an important factor in improving quality of life after injury for an increasing number of SCI patients.

Individually analyzing the reason why patients cannot maintain their physicians' recommended method of bladder management must be done to increase patient compliance. As shown previously, patients informed by a physician about urogenital problems were also informed about the possibility of renal injury and also underwent more recent urologic evaluations, which could help prevent further renal injury.

In conservative cultures like Korea, talking about sexual topics can be taboo. Thus, the actual number of patients with sexual problems may be underestimated. However, sexual activity means not only intercourse or sexual fantasies but also psychological and spiritual relationships, as well as a sense of intimacy with a partner [14]; this characteristic can be difficult for physicians to manage. In the present survey, a large number of SCI patients reported desires for sexual activity, and many of them required supportive methods, although an appropriate method was not recommended. Physicians must be aware of and broach subjects that patients may hesitate to mention first. This could lead to achieving appropriate supportive methods, sex re- 
habilitation, and recovery [14]. Regarding family planning, modern medicine has made having a child through assisted reproduction possible. Nevertheless, we could not determine how many patients preferred this method. Thus, it would be difficult for us to determine any correlation between sexual activity and plans for children.

Through the results of the questionnaire, it was discovered that SCI patients are suffering with many problems related to their quality of life. When managing SCI patients, physicians should show greater concern for problems related with voiding and sexual activity, rather than just physical disability.

\section{SUPPLEMENTARY MATERIAL}

Supplementary material - Questionnaire for spinal cord injury patients - can be found via https://doi.org/10.5213/inj.1630540.270.

\section{REFERENCES}

1. Kim MO. Epidemiology and prognosis of spinal cord injury. In: 2014 Korean Spinal Cord Association review course for spinal cord injury Association; 2014 Aug 9; Seoul, Korea. Bucheon: Korean Spinal Cord Association; 2014.

2. Spinal cord injury facts and figures at a glance [Internet]. Alabama (AL): The National Spinal Cord Injury Statistical Center; c2012 [cited 2014 Aug 1]. Available from: https://www.nscisc.uab.edu/ PublicDocuments/fact_figures_docs/Facts\%202013.pdf.

3. Pershouse KJ, Barker RN, Kendall MB, Buettner PG, Kuipers P, Schuurs SB, et al. Investigating changes in quality of life and function along the lifespan for people with spinal cord injury. Arch Phys Med Rehabil 2012;93:413-9.
4. Cushman DM, Thomas K, Mukherjee D, Johnson R, Spill G. Perceived quality of life with spinal cord injury: a comparison between emergency medicine and physical medicine and rehabilitation physicians. PM R 2015;7:962-9.

5. Definition and Eligibility Criteria 2011-2016 [Internet]. Alabama (AL): The National Spinal Cord Injury Statistical Center; c2005 [cited 2015 Jan 1]. Available from: https://www.nscisc.uab.edu/definition_eligibility.aspx.

6. Shin JC, Kim DH, Yu SJ, Yang HE, Yoon SY. Epidemiologic change of patients with spinal cord injury. Ann Rehabil Med 2013;37:50-6.

7. Yeo JD, Walsh J, Rutkowski S, Soden R, Craven M, Middleton J. Mortality following spinal cord injury. Spinal Cord 1998;36:329-36.

8. Frankel HL, Coll JR, Charlifue SW, Whiteneck GG, Gardner BP, Jamous MA, et al. Long-term survival in spinal cord injury: a fifty year investigation. Spinal Cord 1998;36:266-74.

9. Shavelle RM, DeVivo MJ, Brooks JC, Strauss DJ, Paculdo DR. Improvements in long-term survival after spinal cord injury? Arch Phys Med Rehabil 2015;96:645-51.

10. Strauss DJ, Devivo MJ, Paculdo DR, Shavelle RM. Trends in life expectancy after spinal cord injury. Arch Phys Med Rehabil 2006;87: 1079-85.

11. Oliver M, Inaba K, Tang A, Branco BC, Barmparas G, Schnüriger $\mathrm{B}$, et al. The changing epidemiology of spinal trauma: a 13-year review from a Level I trauma centre. Injury 2012;43:1296-300.

12. Trgovcevic S, Milicevic M, Nedovic G, Jovanic G. Health condition and quality of life in persons with spinal cord injury. Iran J Public Health 2014;43:1229-38.

13. Whalley Hammell K. Quality of life after spinal cord injury: a meta-synthesis of qualitative findings. Spinal Cord 2007;45:124-39.

14. Education and treatment in human sexuality: the training of health professionals. Report of a WHO meeting. World Health Organ Tech Rep Ser 1975;(572):5-33. 
Supplementary material. Questionnaire for spinal cord injury patients

\author{
1. Basic information \\ 1.1 Year of birth \\ 1.2 Sex \\ 1.3 Vector of injury \\ 1.4 Year of injury \\ 1.5 Period of admission \\ 1.6 Number of admissions \\ 1.7 Cohabitation status
}

2. Daily discomforts (choose from listed below)
1) Physical disability
2) Sore
3) Urinary problems
4) Bowel problems
5) Sexual life
6) Pain
7) Hyperreflexia
8) Dyspnea
9) Depression
10) Other

2.1 What is your 'most bothersome' problem? 1st:

2.2 What is the problem you 'wish would improve'? 1st:

2nd: , 2nd: 3rd: 3rd:

3. Daily management of voiding

3.1 Please check the voiding method you are using now, and answer the following questions

3.1.1 Incontinence voiding ( )

3.1.1.1 Managing method

1) Pad or diaper (used per day: )

2) Condom, Latex bag, Gismo

3) Other

3.1.1.2 Additional method

1) Valsalva maneuver

2) Percussion

3) Crede's maneuver

4) Other

3.1.2 Clean intermittent catheterization (CIC) ( )

3.1.2.1 Who does the CIC?

1) Self

2) Family

3) Caregiver

4) Other

3.1.2.2 Which type of catheter do you use? ( )

1) Latex (disposable)

2) Silicon (disposable)

3) Silicon (re-useable), if YES go to 3.1.2.3

4) Other

3.1.2.3 If you are using a re-usable silicon catheter please answer.

Exchange period of catheter: ( ) per month

Exchange period of antiseptic solution: ( ) per month

3.1.3 Urethral Foley catheterization ( )

Exchange period of catheter: ( ) per month 
3.1.4 Suprapubic Foley catheterization ( )

Exchange period of catheter: ( ) per month

3.2 What are the problems related to your current voiding method? (multiple answers allowed)

1) None

2) Incontinence

3) Urinary tract infection

4) Kidney stone

5) Headache or dizziness when bladder is distended

6) Vesicoureteral reflux

7) Other

3.3 Usually where do you get information about urologic management?

1) Physician

2) Spinal cord injury patients

3) Internet, books

4) Other

3.4 When was your most recent urologic examination?

1) Within 1 year

2) Within 3 years

3) Within 5 years

4) Over 5 years ago

5) Never

3.5 What was the recommended method of urinary management by physician?

1) Clean intermittent catheterization

2) Self-voiding

3) Urethral Foley catheterization

4) Suprapubic Foley catheterization

5) Medication

6) Other (multiple answers allowed)

3.6 If you are not on the urinary management your physician recommended, what is the reason?

1) Economic problems

2) No effect

3) Difficult to use catheter

4) No caregiver

5) Side effects

6) Other

3.7 Answer if you are on CIC.

3.7.1 How many times per day?

1) Less than once

2) 1-3 times

3) 3-5 times

4) More than 5 times

5) Varies

3.7.2 Amount of urine per each CIC

1) $100-300 \mathrm{~mL}$

2) $300-500 \mathrm{~mL}$

3) More than $500 \mathrm{~mL}$

3.7.3 What is the difficulty of CIC? (multiple answers allowed)
1) Difficult to use
2) Economic problem
3) Side effects
4) Difficult to find places for CIC
5) Hard to keep sanitized
6) Other 
3.7.4 What is the side effect of CIC? (multiple answers allowed)

1) Urinary tract infection

2) Urethral stricture

3) Headache, dizziness

4) Pain

5) Bladder stone

6) Incontinence

7) Other

3.8 Have you had any of the procedures/operations below? (multiple answers allowed)

1) Bladder augmentation

2) Artificial urethral sphincter implantation

3) Bulking agent for urethra

4) Urinary bypass

5) Incision of the sphincter

6) Dialysis

7) Anti-reflux surgery

8) Other

3.9 Do you know of the possibility of renal injury due to bladder dysfunction?

1) Yes (Go to 3.10)

2) No

3.10 What was your source of information? (multiple answers allowed)

1) Physician

2) Spinal cord injured colleagues

3) Internet or books

4) Other

3.11 What was your expense for urinary management in 2013?

Per month: KRW (Korean won)

3.12 Are you taking voiding related medications?

1) Yes

2) No

4. Sex and family planning

4.1 Do you have children?

1) Yes

2) No

4.2 Do you plan to have children?

1) Yes

2) No

4.3 Do you have a sex life?

1) Yes

2) No

4.4 Are you satisfied with your current sex life?

1) Very unsatisfied

2) Quite unsatisfied

3) Average

4) Quite satisfied

5) Very unsatisfied 
4.5 What is your complaint about your present sex life? (multiple answers allowed)

1) Lack of libido

2) Impotence

3) Able to achieve erection but unable to go through with intercourse

4) Able to go through with intercourse but cannot last long

5) No partner

6) Other

4.6 Do you have a sexual desire?

1) None

2) Slight

3) Average

4) Quite a lot

5) Extreme

4.7 What was the recommended supportive method from the physician for sexual activity? (multiple answers allowed)

1) Medication

2) Penile injection of drug

3) Suppository of drug

4) Vacuum constriction device

5) Penile prosthetic implantation

6) Cream

7) None

8) Other

4.8 Which supportive method are you using currently? (multiple answers allowed)

1) Medication

2) Penile injection of drug

3) Suppository of drug

4) Vacuum constriction device

5) Penile prosthetic implantation

6) Cream

7) None

8) Other

4.9 If you are not using a physician's recommended method, what is the reason? (multiple answers allowed)
1) No effect
2) Financial problems
3) Pain
4) Side effects
5) Other 